\title{
Rescue thrombolysis partial failure in massive PE complicated with in-transit thrombus
}

This article was published in the following Dove Medical Press journal: International Medical Case Reports Journal

\author{
Toktam Alirezaei' \\ Zahra Ansari Aval ${ }^{2}$ \\ 'Cardiology Department of Shohaday- \\ e-Tajrish Hospital, Shahid Behesti \\ University of Medical Science, Tehran, \\ Iran; ${ }^{2}$ Cardiovascular Research Center, \\ Shahid Behesti University of Medical \\ Science, Tehran, Iran
}

\begin{abstract}
A 58-year-old man who presented with syncope, dyspnea, and hemodynamic compromise was found to have large free-floating right atrial thrombuses on echocardiogram. Decision was made to transfer the patient for emergent atriotomy. Cardiothoracic surgeons declared the patient as inoperable and recommended to use a lytic agent. Alteplase was administered with subsequent near-complete resolution of symptoms and near-normalization of echocardiographic parameters. The post-thrombolytic course was complicated by saddle pulmonary emboli requiring embolectomy. Catheter embolectomy was not available and cardiothoracic surgeon in other center considered the patient to be very high risk for transferring between hospitals and surgical intervention. Ultimately, the critical decision was made, despite the patient having been administered thrombolytic therapy within the previous 48 hours. Alteplase was given, but was not effective and the patient required surgical intervention. Surgical embolectomy was done successfully in another hospital and the patient was discharged with warfarin.
\end{abstract}

Keywords: right atrial thrombus, pulmonary embolism, rescue thrombolysis, surgical embolectomy

\section{Introduction}

Large free-floating right atrial (RA) thrombus represents a rare condition that is associated with high rates of morbidity and mortality; also, it can complicate with acute pulmonary thromboembolism. Although it is a critical setting and a major therapeutic emergency, no definite guideline recommendations have been suggested for this particular condition and the treatment should be conducted based on experience or empirical evidence. In such cases, anticoagulation with heparin, thrombolytic therapy, percutaneous catheter intervention, or surgical embolectomy are considered possible therapeutic options. ${ }^{1-4}$

It is common for clinicians to be faced with the dilemma of how to manage a mobile right heart thrombus in life-threatening settings. In the present case, the problem was complicated subsequently by post-thrombolytic acute pulmonary thromboembolism, requiring intervention.

\section{Case presentation}

A 58-year-old male was emergently brought to our hospital with sudden episode of syncope at home. The patient reported to have experienced breathlessness during the past 5 days before admission. Breathlessness was progressive in nature from $\mathrm{New}$ York Heart Association grade 2-4. He also had complained of recurrent vomiting and inability to take food since two days. 
The patient had a 15 pack-year smoking history. Medical history and family history were not contributory.

On initial evaluation, his vital signs showed an systolic blood pressure of $70 \mathrm{mmHg}$, a heart rate of 110 beats per minute, a respiratory rate of 34 per minute, and oxygen saturation of $88 \%$ in ambient air. Jugular veins were distended.

Electrocardiogram at rest showed sinus rhythm $100 \mathrm{bpm}$, S1Q3T3 pattern, and negative T waves in leads V1-V4.

The laboratory findings were all within normal limits and nothing specific was found on the laboratory tests except creatinine of $3.25 \mathrm{mg} / \mathrm{dL}$. The chest radiograph showed enlarged central pulmonary arteries and diminished peripheral pulmonary vascular marking with no active lung lesions (Figure 1). The patient had no symptom or sign of deep vein thrombosis and did not undergo a follow-up imaging study for evaluation of venous system.

Because of the high pretest probability for pulmonary embolism, a two-dimensional echocardiogram was performed in the emergency department (Video S1). It showed severely dilated RA, right ventricle (RV), inferior vena cava, and severely elevated right ventricular systolic pressure ( $\mathrm{PAP}=70 \mathrm{mmHg}$ ). The RV function was markedly depressed. Additionally, large free-floating thrombus was seen in the right atrium, protruding into the RV during diastole.

Based on the diagnosis of RA thrombus (likely with concomitant pulmonary emboli $[\mathrm{PE}]$ ) and hemodynamic compromise, surgical intervention was considered.

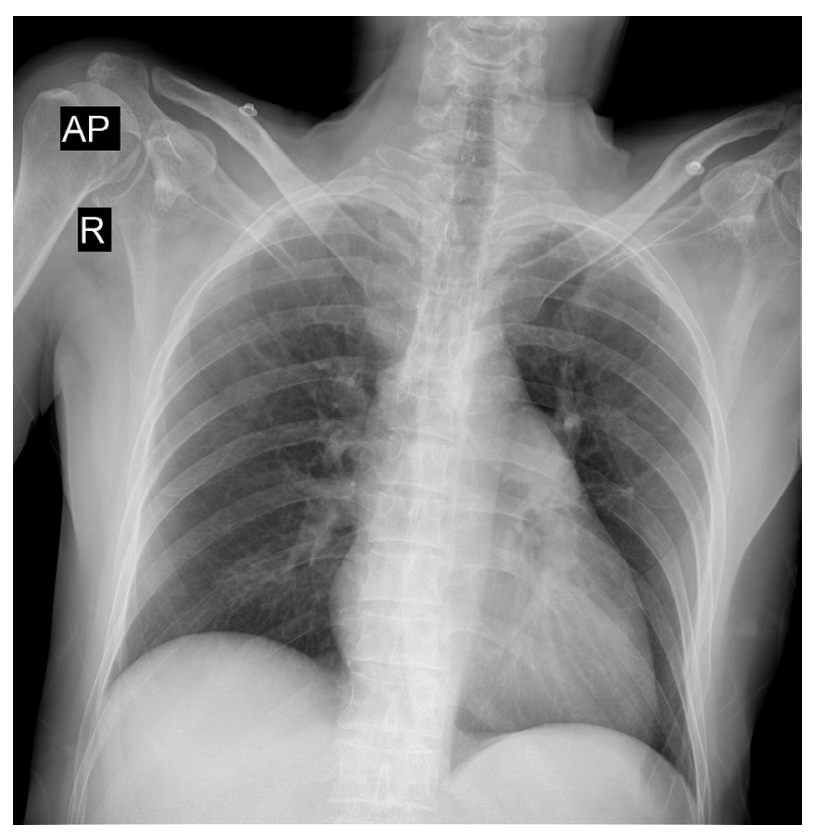

Figure I Chest radiograph demonstrating enlarged central pulmonary arteries and diminished peripheral pulmonary vascular marking. Abbreviations: AP, anterior-posterior; R, right.
Because of lack of surgical expertise in our center, surgical consult with cardiothoracic surgeon in another center was requested but because of the critical condition of the patient, complicated by unstable hemodynamic parameters, the surgery team declared the patient as inoperable and recommended use of a lytic agent.

Ultimately, despite the fear of lysing the RA thrombus that was alone sufficient to cause embolization to the RV and pulmonary arteries, the decision was made to treat the patient with systemic thrombolysis.

The patient was thrombolyzed with $100 \mathrm{mg}$ infusion over a 2-hour period of alteplase. Then the patient was started with an intravenous drip of unfractionated heparin.

Approximately 1 hour after the thrombolysis treatment, significant clinical improvement was seen. BP was $85 / 55$ $\mathrm{mmHg}$, with a heart rate of 90 beats per minute and a respiratory rate of 20 per minute with an oxygen saturation of $94 \%$ in ambient air. A repeat transthoracic echocardiogram 12 hours after the administration of thrombolytic agent showed complete resolution of the thrombus, significant decrease in size of the RA, RV and inferior vena cava, RV function improvement, and reduction in pulmonary artery pressure ( $\mathrm{PAP}=45 \mathrm{mmHg}$ ).

The patient experienced low urine output and the serum creatinine was increased to $4.5 \mathrm{mg} / \mathrm{dL}$.

Low urine output and hypotension state were considered due to hypovolemia and dehydration, which require fluid boluses and could be corrected by volume resuscitation.

So, he continued to receive fluid boluses. Urine output increased to $>0.5 \mathrm{~mL} / \mathrm{kg} /$ hour and serum creatinine decreased to $1.5 \mathrm{mg} / \mathrm{dL}$.

Despite fluid resuscitation, he remained hypotensive with a systemic BP of $85 / 60$, requiring intravenous norepinephrine.

Nearly after 40 hours of admission, the patient was tachypneic with continued hypotension and he had hypoxia with an oxygen saturation of $87 \%$.

Transthoracic echocardiography was done again (Figure 2), it revealed right heart dilatation, RV compressing the left ventricle, RV free wall hypokinesia, right ventricular systolic pressure $-60 \mathrm{mmHg}$ - all of which were indirect aspects of pulmonary embolism following lysis of RA clot.

Pulmonary artery CT angiography affirmed large bilateral pulmonary thromboemboli (Figure 3).

According to the diagnosis of massive pulmonary embolism and treatment with thrombolytic agent less than 48 hours ago, the patient became candidate for emergent catheter embolectomy and he had an emergent consult to vascular 

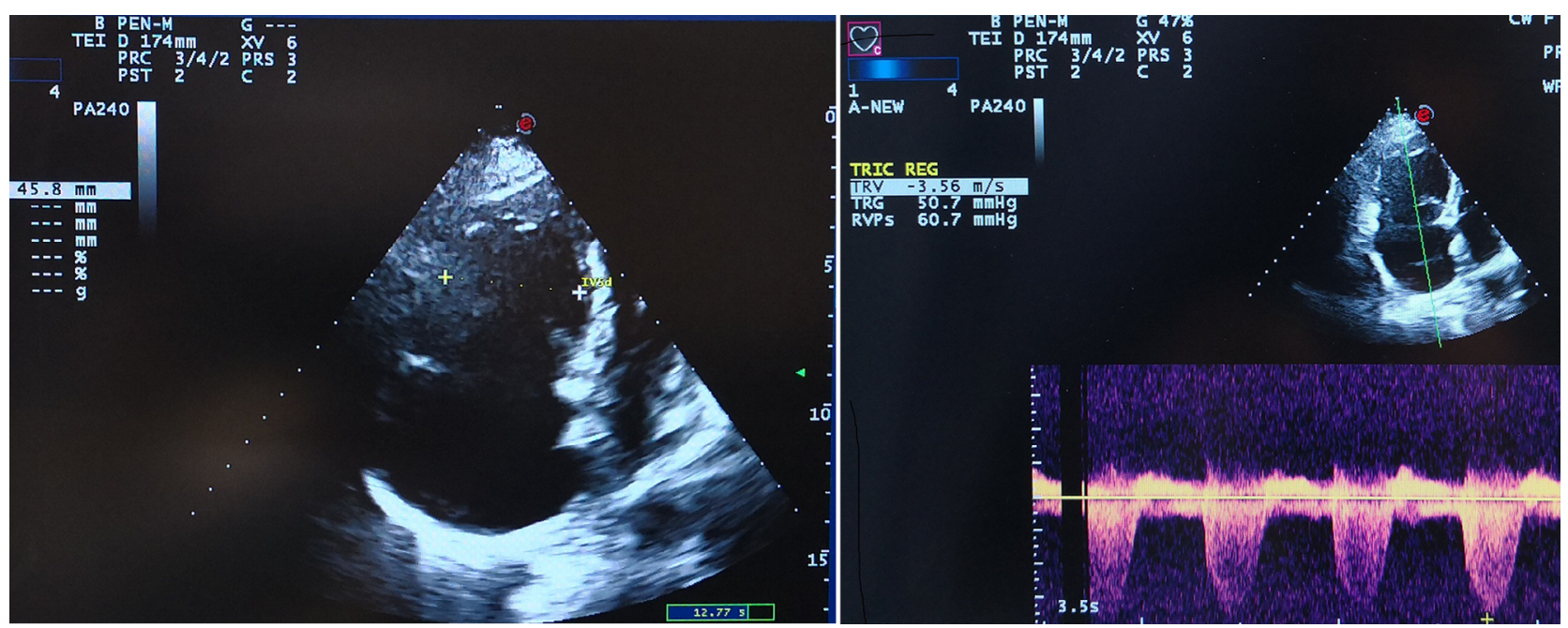

Figure 2 Transthoracic echocardiogram showing (left) right heart dilatation and (right) pulmonary hypertension.
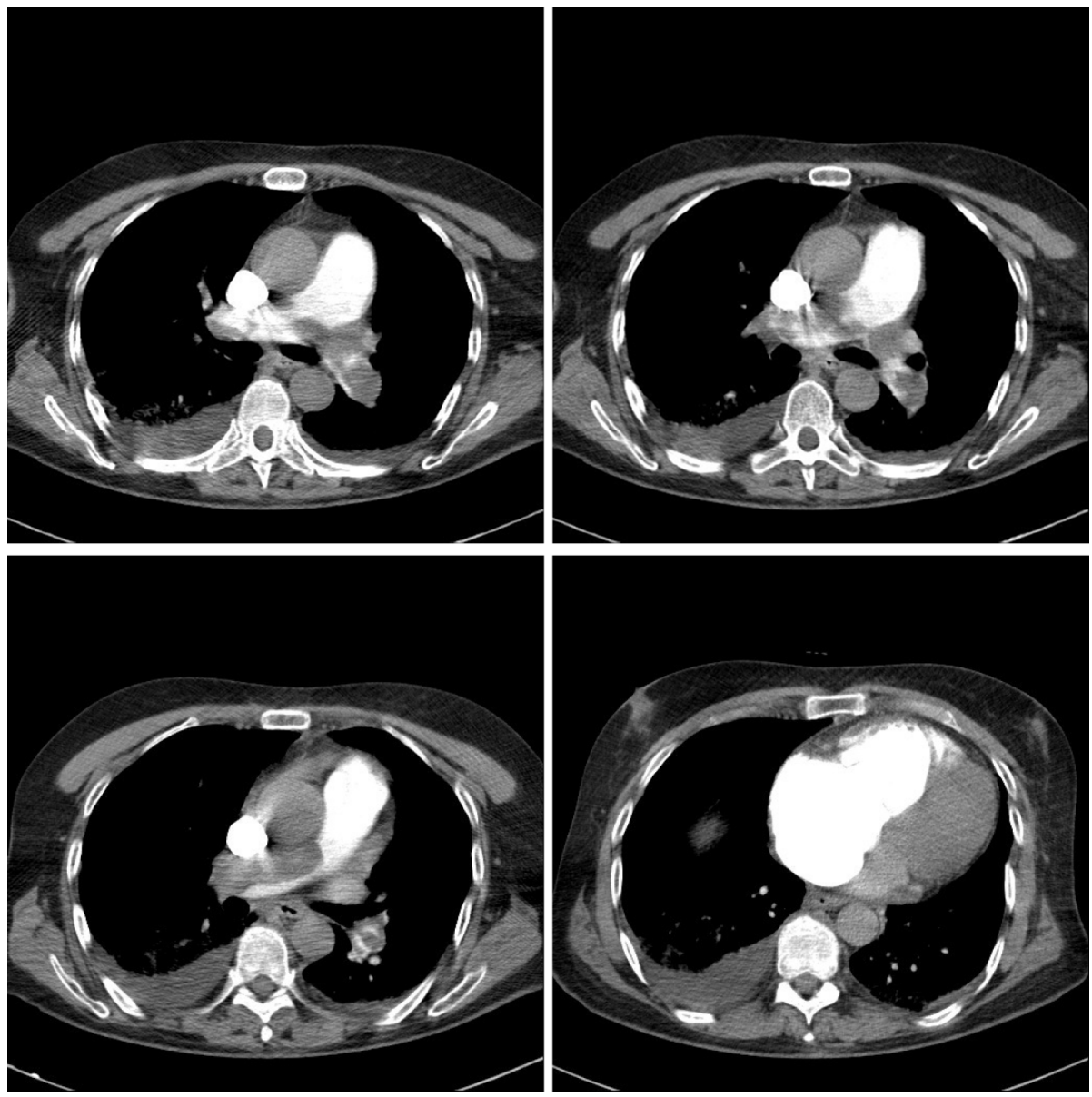

Figure 3 Pulmonary CT angiography demonstrates bilateral saddle pulmonary thromboemboli with right ventricle dilatation.

surgery. But due to unavailability of equipment for catheter embolectomy, vascular surgery team recommended surgical embolectomy. Surgical consult with additional acardiothoracic surgeons was requested and they considered the patient too much of a high risk for surgical intervention and recommended medical therapy.

Thus, based on surgeon opinion, the patient's critical condition and multidisciplinary assessment, a critical decision 
was made to treat the patient with systemic thrombolysis, therefore, alteplase over 2 hours at a dose of $100 \mathrm{mg}$, once again, was administered.

After the administration of systemic thrombolytic, the patient continued symptoms and a repeat transthoracic echocardiogram showed no change in echocardiographic findings.

At this time, his vital signs showed an SBP of $70 \mathrm{mmHg}$, a heart rate of 96 beats per minute, a respiratory rate of 30 per minute, and oxygen saturation of $82 \%$ in ambient air.

Because of no response to thrombolytic therapy, although the patient was considered very high risk for transferring between hospitals and the risk of bleeding following thrombolytic treatment was very high, he was transferred to another center for surgical embolectomy. The patient underwent surgery on 1 day after thrombolytic administration. Surgery was successful and large clots were removed from the pulmonary artery system (Figure 4).

Afterward, the patient was given heparin anticoagulation and warfarin. The patient was weaned from bypass and the remainder of the hospital course was not complicated. He was discharged and was advised to continue warfarin with repeated international normalized ratio (INR) testing intermittently. During 3-month follow-up and review, the patient was asymptomatic with the INR maintained between 2.0 and 3.0 with warfarin.

\section{Ethics statement}

We confirm that any aspect of the work covered in this manuscript that has involved experimental human patient has been

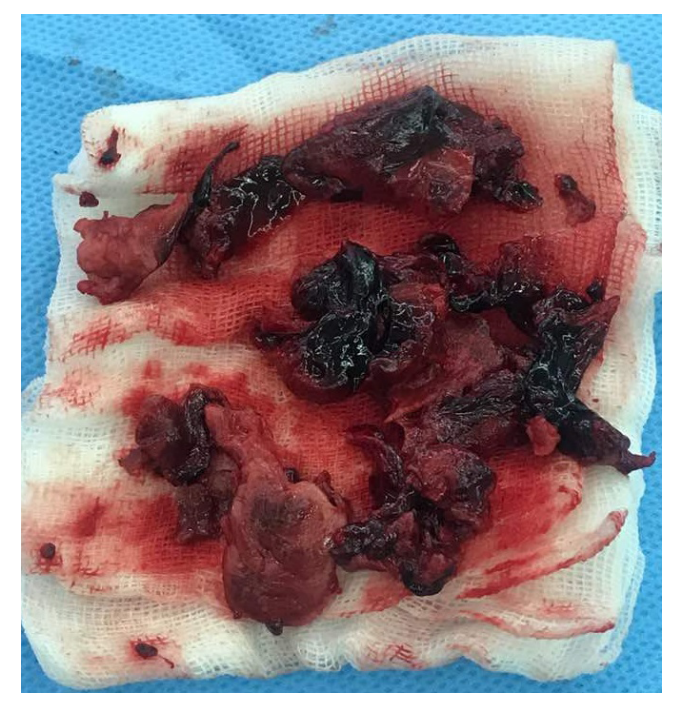

Figure 4 Thrombuses removed from the left and right pulmonary arteries at the time of surgery. conducted with the ethical approval of all relevant bodies and that such approvals are acknowledged within the manuscript. Written informed consent for publication of his clinical details, clinical images, and videos was obtained from the patient. Institutional approval was not required for this manuscript.

\section{Discussion}

The unexpected appearance of an RA thrombus on twodimensional echocardiogram often is a surprise to the clinician.

RA thrombus is an uncommon, but life-threatening, a condition that is associated with a high mortality rate. The results of previous studies showed the rate of mortality up to $100 \%$ with no treatment and $45 \%$ with treatment. ${ }^{2,5}$

RA thrombus is a challenge for treatment. Yet, there is a dilemma that when the diagnosis of RA thrombus (with or without concomitant $\mathrm{PE}$ ) has been affirmed, what approach constitutes optimal management. The number of studies in previously treated patients have revealed that the optimal treatment, among patients with no contraindications to thrombolysis, is to use a lytic factor in an effort to dissolve the RA thrombus in situ and successful thrombolysis was associated with an improved survival when compared either with surgery or anticoagulation treatment. ${ }^{6}$

Rose et al reported the mortality experience for thrombolysis, surgical embolectomy, and anticoagulation therapy to be $11.3 \%, 23.8 \%$, and $28.6 \%$, respectively. ${ }^{2}$ Despite this, there clear guidelines to prescribe a definite recommendation are lacking and the guidelines failed to reach a clear therapeutic strategy based on retrospective case studies. ${ }^{7,8}$

In this study, we present a case of a large free-floating thrombus of the RA, which was treated with thrombolytic agent. From the beginning of the thrombolysis and afterward, there was fear that lysing of large RA thrombus caused embolization to the pulmonary arteries. For this reason, before the start of thrombolytic treatment, we attempted to transfer the patient for surgery. Finally, the patient received thrombolytic therapy and it appeared to be successful based on the significant clinical improvement, disappearance of the RA thrombus and signs of RV overload. However, the patient remained hypotensive and required inotrope support despite fluid resuscitation. Eventually, recurrent massive PE was identified after 2 days.

Systemic thrombolysis in the setting of RA thrombus is associated with a considered risk of clot fragmentation and migration, extensive embolization to pulmonary arteries, or repeated PE following partial resolution of the venous 
thrombus. ${ }^{5}$ In our patient, persistent hypotension and large clots in CT angiography suggested that the most probable mechanism for recurrent $\mathrm{PE}$ after thrombolysis was embolism of the fragmented RA thrombus into the pulmonary arteries.

The major challenge for us was how to manage the postthrombolytic PE.

Much less is known about the optimal management approach for this difficult clinical situation.

According to the possible mechanism for recurrent PE, the first decision was to request a surgical consult for embolectomy. As mentioned previously, surgical intervention was not executable at that time and hence we had to administer thrombolytic agent again.

The stenosis resulting from rethrombosis offers a thrombogenic surface and grows slowly until the vessel becomes completely obliterated, in this setting, where pulmonary arteries are packed with dense thrombotic material, thrombus resistance and rescue thrombolysis failure is possible.

We were not sure of the safety and efficacy of repeat thrombolysis but it was the only available treatment option and we have experienced successful results from thrombolytic treatment in our previous cases. ${ }^{9,10}$ So, rescue thrombolysis was used only as a last resort for life saving and not as a standard step or current procedure. Unfortunately, in this case, the patient was not responsive to thrombolysis; however, surgical embolectomy could be life-saving and should be performed despite the high risk of surgery.

Debate continues about the best approach for patients with RA thrombus at risk for post-thrombolytic PE. There is no consensus among cardiologists and surgical specialists about the optimal management in these patients, but it is necessary to prevent treatment complications, improve clinical outcomes, and as a result, provide the best life-saving strategy for the patients.

This case report also highlights the need and importance of regular follow-up following thrombolytic therapy to prevention of treatment-related problems. Greco et $\mathrm{al}^{5}$ evaluated thrombus behavior during administration of alteplase by two-dimensional echocardiogram; they reported the lysis occurred with a progressive fragmentation in little particles that move from the heart chambers to pulmonary arteries to eventually disappear. However, when reviewing the available literature, it seems that it is unclear whether the disappearance of right heart thrombosis following thrombolytic treatment is due to complete dissolving of the thrombus or migration to the pulmonary circulation. ${ }^{11}$

The most effective and safe therapy for patients with a large RA thrombus remains unknown. Previous studies, regardless of the size of the atrial thrombus, have recom- mended thrombolytic therapy rather than surgery. ${ }^{2,4}$ This case report suggests that the size of the thrombus should be considered in selection of the management strategy, because the fragmented atrial thrombus might be the cause of a sudden pulmonary embolism and the risk of fragmented thrombusinduced PE appears to be related to the large size of the thrombus. It seems that surgery would be selected more often when the size of the right heart thrombus is large. Although, the surgical embolectomy has potential complications, including general anesthesia, cardiopulmonary bypass, and an inherent delay time, but a post-thrombolytic PE appears to carry a poor prognosis. ${ }^{12}$

Finally, it is necessary to conduct controlled trials to evaluate the efficacy of any treatment and determine the safety parameters to prevent treatment complications.

\section{Disclosure}

The authors report no conflicts of interest in this work.

\section{References}

1. Konstantinides SV, Torbicki A, Agnelli G, et al. 2014 ESC guidelines on the diagnosis and management of acute pulmonary embolism. Eur Heart J. 2014;35(43):3033-3069.

2. Rose PS, Punjabi NM, Pearse DB. Treatment of right heart thromboemboli. Chest. 2002;121(3):806-814.

3. Narang A, Mediratta A, Estrada JR, et al. Transcatheter therapy for a large mobile right atrial thrombus and massive pulmonary embolism. J Invasive Cardiol. 2016;28(5):E49-E51.

4. Goldhaber SZ. Optimal strategy for diagnosis and treatment of pulmonary embolism due to right atrial thrombus. Mayo Clin Proc. 1988;63(12):1261-1264.

5. Greco F, Bisignani G, Serafini O, Guzzo D, Stingone A, Plastina F. Successful treatment of right heart thromboemboli with IV recombinant tissue-type plasminogen activator during continuous echocardiographic monitoring: a case series report. Chest. 1999;116(1):78-82.

6. Mancuso L, Marchì S, Mizio G, Iacona MA, Celona G. Echocardiographic detection of right-sided cardiac thrombi in pulmonary embolism. Chest. 1987;92(1):23-26.

7. Chapoutot L, Nazeyrollas P, Metz D, et al. Floating right heart thrombi and pulmonary embolism: diagnosis, outcome and therapeutic management. Cardiology. 1996;87(2):169-174.

8. Kinney EL, Wright RJ. Efficacy of treatment of patients with echocardiographically detected right-sided heart thrombi: a meta-analysis. Am Heart J. 1989;118(3):569-573.

9. Alirezaei T, Hajimoradi B, Pishgahi M, Nekooghadam SM, Golmohamadi M. Successful systemic thrombolytic therapy for massive pulmonary embolism in a patient with breast cancer, brain metastasis, and thrombocytopenia: a case report. Clin Case Rep. 2018;6(8):1431-1435.

10. Pishgahi M, Alirezaei T, Hajimoradi B. Systemic fibrinolytic therapy in the presence of absolute contraindication; a case series. Emergency J. 2018;6(1):e25.

11. Patel AK, Kafi A, Bonet A, et al. Resolution of a mobile right atrial thrombus complicating acute pulmonary embolism with low-dose tissue plasminogen activator in a patient with recent craniotomy. J Intensive Care Med. 2016;31(9):618-621.

12. Aymard T, Kadner A, Widmer A, et al. Massive pulmonary embolism: surgical embolectomy versus thrombolytic therapy-should surgical indications be revisited? Eur J Cardiothorac Surg. 2013;43(1): 90-94. 
International Medical Case Reports Journal

Dovepress

Publish your work in this journal

The International Medical Case Reports Journal is an international, peer-reviewed open-access journal publishing original case reports from all medical specialties. Previously unpublished medical posters are also accepted relating to any area of clinical or preclinical science. Submissions should not normally exceed 2,000 words or

4 published pages including figures, diagrams and references. The manuscript management system is completely online and includes a very quick and fair peer-review system, which is all easy to use. Visit http://www.dovepress.com/testimonials.php to read real quotes from published authors.

Submit your manuscript here: https://www.dovepress.com/international-medical-case-reports-journal-journal 